\title{
Phrenic Nerve Blockade to Diagnose and Treat Diaphragmatic Pain After Surgical Repair of Congenital Diaphragmatic Eventration
}

\author{
Sujana Dontukurthy, e, Saptarshi Biswas ${ }^{\mathrm{b}}$, Sudhakar Kinthala ${ }^{\mathrm{c}}$, \\ Waala Housny ${ }^{\mathrm{d}}$, Joseph D. Tobias ${ }^{\mathrm{a}}$
}

\begin{abstract}
A 46-year-old woman presented with pain in the lateral side of the left chest wall and a sensation of fullness and pulling in the epigastric region, which started 4 weeks following diaphragmatic plication for left diaphragmatic eventration. The patient was diagnosed as suffering from post-thoracotomy pain syndrome (PTPS). A diagnostic intercostal nerve block relieved the chest wall pain, but not the epigastric pain. After a detailed evaluation, the epigastric pain was postulated to be of diaphragmatic origin and hence a diagnostic phrenic nerve block was performed which relieved the epigastric pain. Combined intercostal nerve neurolysis and phrenic nerve block relieved her pain completely. The phrenic nerve may play a role in pain transmission and the genesis of chronic pain following diaphragmatic surgery. Diaphragmatic pain following surgery may contribute to the development of chronic pain. Phrenic nerve blockade provides diagnostic information regarding the etiology of pain as well as being effective in providing analgesia. The technique of phrenic nerve block is presented and its role in the diagnosis and treatment of pain following thoracic surgery is reviewed.
\end{abstract}

Keywords: Diaphragmatic plication; Thoracic surgery; Diaphragmatic eventration; Chronic pain; Post-thoracotomy pain syndrome; Intercostal nerve block; Phrenic nerve block; Diaphragmatic pain

\section{Introduction}

Thoracotomy is associated with a high incidence of post-thora-

Manuscript submitted February 5, 2020, accepted April 1, 2020

aDepartment of Anesthesiology and Pain Medicine, Nationwide Children's Hospital, Columbus, OH 43205, USA

bDepartment of Surgery, Forbes Hospital, Allegheny Health Network, Monroville, PA 15146, USA

'Department of Anesthesiology and Pain Medicine, Guthrie Robert Packer Hospital, Sayre, PA 18840, USA

dDepartment of Anesthesiology and Pain Medicine, Brookdale University Medical Center, Brooklyn, NY 11212, USA

${ }^{e}$ Corresponding Author: Sujana Dontukurthy, Department of Anesthesiology and Pain Medicine, Nationwide Children's Hospital, Columbus, OH 43205, USA. Email: Sujana.Dontukurthy@nationwidechildrens.org

doi: https://doi.org/10.14740/jmc3436 cotomy pain syndrome (PTPS), which may be debilitating and interfere with performance of routine daily activities. The incidence of PTPS may be approximately $10-50 \%[1,2]$. Apart from PTPS, patients may present with pain of different origins and hence a thorough assessment is required prior to the establishment of correct diagnosis. In this report, we describe a patient who presented with PTPS along with an abnormal fullness and pulling sensation in the epigastric region following diaphragmatic plication for congenital diaphragmatic eventration. The PTPS was relieved by intercostal nerve blockade. However, the pain and sensations in the epigastric region were not relieved and hence it was postulated to be arising from the diaphragm. A diagnostic phrenic nerve block was performed which significantly relieved the pain. To our knowledge, this is the only report of diaphragmatic pain after diaphragmatic eventration repair diagnosed and relieved by phrenic nerve blockade.

\section{Case Report}

A 46-year-old woman presented with shortness of breath, food intolerance, and inability to sleep in the supine position for 1.5 years. The patient was known to have an elevated left hemidiaphragm diagnosed as diaphragmatic eventration since her childhood, which was confirmed by chest radiograph and computed tomography (CT) scan. Diaphragmatic plication was performed with a $10-\mathrm{cm}$ low lateral thoracotomy incision at the ninth intercostal space. The postoperative course was uneventful. The patient presented 1 year later, complaining of pain in the left lateral chest wall and left abdominal upper quadrant along with a sensation of fullness in the epigastric region which started 4 weeks after the surgery. The pain was described as sharp and stabbing exacerbated by movement and local touch, which was rated as $7 / 10$ on visual analogue scale (VAS) on the lateral chest wall. The sensation of fullness in the epigastric region was aggravated by the upright posture, food intake and bending forwards rather than coughing or deep breathing. The nature of the pain was described as a pulling pain, rated as $8 / 10$ on the VAS. There was no history of shortness of breath, cough or chest pain. On examination, the patient had significant hyperalgesia and hyperesthesia covering a large area of the lateral left chest wall from the midaxillary line to the left upper quadrant in the distribution of the 
thoracotomy scar. The incision scar was clean and well healed. No tenderness or mass was elicited at the site of epigastrium. Chest radiograph and upper gastrointestinal endoscopy were obtained to exclude the possibility of recurrence of the eventration and injury to the abdominal viscera. Radiologic imaging was unremarkable and showed the repair to be intact, with no visceral injury or neuromas identified at the incision site. The patient had earlier received an intercostal block which provided partial pain relief. Prior medical treatment included non-steroidal anti-inflammatory drugs (NSAIDS) and gabapentin which provided only partial pain relief.

A diagnosis of PTPS was made and left-sided diagnostic intercostal nerve blocks at the ninth to 11th thoracic intercostal spaces were performed. Pain was relieved only in the thoracotomy scar area, and the patient complained of persistent epigastric discomfort. The patient returned to her home country, but presented with the same complaint 1 year later. A repeat left diagnostic intercostal nerve block was performed in preparation for a possible intercostal neurolysis. Again, the thoracic wall pain improved but the epigastric discomfort persisted. As the earlier blocks proved ineffective in eliminating the epigastric discomfort and multiple investigations were unremarkable, involvement of the phrenic nerve was postulated as the cause of the discomfort. With a history of left diaphragmatic paralysis, a diagnostic left phrenic nerve block was planned. Under ultrasound guidance, the left phrenic nerve was identified under the left sternocleidomastoid muscle and above scalenus anterior muscle. Using a 22 -gauge echogenic needle, a total of $10 \mathrm{~mL}$ of $0.5 \%$ ropivacaine was injected to anesthetize the left phrenic nerve. There was significant improvement of the abdominal pain and fullness following the left phrenic nerve block. The patient still had some residual pain along the chest wall radiating to lateral and anterior abdominal wall. It was postulated that the pain was mediated by two separate sites, including the intercostal nerves due to intercostal neuralgia at the incision site, and due to traction of the peritoneal ligaments of the stomach on the site of repair at the diaphragm mediated by the left phrenic nerve. A combined ultrasound-guided left intercostal neurolytic block for left intercostal neuralgia and local anesthetic phrenic nerve block for her epigastric pain was planned. Under ultrasound guidance with real time imaging, $15 \mathrm{~mL}$ of $0.6 \%$ phenol was injected in the ninth to 11 th thoracic intercostal spaces. Ultrasound-guided phrenic nerve blockade was also performed with $5 \mathrm{~mL}$ of $0.5 \%$ ropivacaine and methylprednisolone acetate $(40 \mathrm{mg})$. As there is a paucity of literature on phrenic nerve neurolysis, a corticosteroid was added to the local anesthetic for the blockade with the intent to increase the duration of block. The patient experienced significant pain relief with a decrease of the VAS from $8 / 10$ to $3 / 10$. The pain relief lasted for $4-6 \mathrm{~h}$. The result was confirmed with a repeat phrenic nerve block at a later date that also resulted in a complete pain relief for a similar duration. In both instances, the patient had a transient partial sensorimotor blockade of the left shoulder, which is an expected consequence of the phrenic nerve block.

\section{Discussion}

PTPS is defined by the International Association for the Study of Pain (IASP) as "pain that recurs or persists along a thoracotomy incision at least 2 months following the surgical procedure" [3]. Although the use of minimally invasive surgeries may decrease the incidence of PTPS, the incidence of longterm persistent pain has also been reported to be the same after both open and minimally invasive procedures [4-7]. The pathogenesis of PTPS is complex. It is proposed that the input from the somatic pain receptors is conducted through the intercostal nerves to the dorsal horn, while the phrenic nerve transmits noxious stimuli from the diaphragm, mediastinum, and pericardial pleura [8]. The neuropathic pain has been attributed to direct nerve injury at the time of surgery or nerve entrapment from scar tissue formation [2, 6, 8]. Non-abdominal etiologies of abdominal pain such as pleural and diaphragmatic pathologies can also present as abdominal pain.

PTPS may result after poorly controlled acute postoperative pain and tends to be more common in females than males $[9,10]$. While a thorough assessment is required to exclude pathology from other sources that can mimic thoracotomy pain, intercostal nerve blockade can be used to confirm the diagnosis of intercostal neuralgia. The severity of PTPS can range from mild to severe. PTPS may respond to pharmacologic treatments including NSAIDS, topical application of capsaicin, and neuromodulators such as gabapentin [11]. Invasive treatment modalities including intercostal nerve blockade, epidural analgesia, and spinal cord stimulation may be necessary if pain is resistant to pharmacologic treatments $[12,13]$.

In our patient, apart from the lateral thoracic pain which was relieved after by intercostal nerve blockade, she also experienced fullness and a pulling sensation in the abdomen even after medical treatment and intercostal nerve blockade. This abnormal sensation was relieved after left phrenic nerve blockade.

Phrenic nerve blockade has been used to treat intractable hiccups when conservative and pharmacologic treatment is unsuccessful and to provide analgesia during percutaneous needle biopsy of the lung $[14,15]$. However, phrenic nerve blockade for the treatment of diaphragm-mediated pain presenting as abdominal pain has not been previously described. Incomplete pain relief after intercostal blockade led to the recognition of another component of our patient's pain, which required a secondary evaluation for the etiology of pain and its subsequent treatment.

This case report highlights the fact that multiple nerves may be involved in the genesis of chronic pain including PTPS. A thorough assessment using multiple regional anesthetic techniques may be required to identify and treat the etiology of the pain. Phrenic nerve blockade may be a useful technique to diagnose and treat diaphragmatic pain, and should be considered both as a diagnostic and therapeutic option in the management of abnormal epigastric or abdominal pain that does not respond to other interventions, especially following surgery involving the diaphragm.

\section{Acknowledgments}

None to declare. 


\section{Financial Disclosure}

None to declare.

\section{Conflict of Interest}

None to declare.

\section{Informed Consent}

Written informed consent was obtained from the patient for the procedure.

\section{Author Contributions}

Sujana Dontukurthy: concept, review of the case, preparation of initial drafts and final version. Saptarshi Biswas: review of case, and preparation of final version. Sudhakar Kinthala: review of case, and preparation of final version. Walaa Housny: review of case, and preparation of final version. Joseph D. Tobias: review of case, preparation of initial drafts and final version.

\section{Data Availability}

The authors declare that data supporting the findings of this study are available within the article.

\section{References}

1. Kehlet H, Jensen TS, Woolf CJ. Persistent postsurgical pain: risk factors and prevention. Lancet. 2006;367(9522):1618-1625.

2. Karmakar MK, Ho AMH. Post thoracotomy pain syndrome. Thorac Surg Clin. 2004;14:345-352.

3. Merskey H. Classification of chronic pain: Description of chronic pain syndromes and definitions of pain terms. Pain. 1986;3:S138-139.
4. Maguire MF, Ravenscroft A, Beggs D, Duffy JP. A questionnaire study investigating the prevalence of the neuropathic component of chronic pain after thoracic surgery. Eur J Cardiothorac Surg. 2006;29(5):800-805.

5. Forster R, Storck M, Schafer JR, Honig E, Lang G, Liewald F. Thoracoscopy versus thoracotomy: a prospective comparison of trauma and quality of life. Langenbecks Arch Surg. 2002;387(1):32-36.

6. Perttunen K, Tasmuth T, Kalso E. Chronic pain after thoracic surgery: a follow-up study. Acta Anaesthesiol Scand. 1999;43(5):563-567.

7. Furrer M, Rechsteiner R, Eigenmann V, Signer C, Althaus $\mathrm{U}$, Ris HB. Thoracotomy and thoracoscopy: postoperative pulmonary function, pain and chest wall complaints. Eur J Cardiothorac Surg. 1997;12(1):82-87.

8. Hazelrigg SR, Cetindag IB, Fullerton J. Acute and chronic pain syndromes after thoracic surgery. Surg Clin North Am. 2002;82(4):849-865.

9. Erdek M, Staats PS. Chronic pain after thoracic surgery. Thorac Surg Clin. 2005;15:123-130.

10. Gotoda Y, Kambara N, Sakai T, Kishi Y, Kodama K, Koyama T. The morbidity, time course and predictive factors for persistent post-thoracotomy pain. Eur J Pain. 2001;5(1):89-96.

11. Sihoe AD, Lee TW, Wan IY, Thung KH, Yim AP. The use of gabapentin for post-operative and post-traumatic pain in thoracic surgery patients. Eur J Cardiothorac Surg. 2006;29(5):795-799.

12. de Leon-Casasola OA. Spinal cord and peripheral nerve stimulation techniques for neuropathic pain. J Pain Symptom Manage. 2009;38(2 Suppl):S28-38.

13. Graybill J, Conermann T, Kabazie AJ, Chandy S. Spinal cord stimulation for treatment of pain in a patient with post thoracotomy pain syndrome. Pain Physician. 2011;14(5):441-445.

14. Renes SH, van Geffen GJ, Rettig HC, Gielen MJ, Scheffer GJ. Ultrasound-guided continuous phrenic nerve block for persistent hiccups. Reg Anesth Pain Med. 2010;35(5):455-457.

15. Carrero E, Arguis P, Sanchez M, Sala-Blanch X. Ultrasound-guided phrenic nerve block for CT-guided percutaneous pulmonary fine-needle aspiration biopsy. J Vasc Interv Radiol. 2015;26(4):597-599. 